\title{
Recherches sur les circuits commerciaux du nord de l'Espagne à la fin du Moyen Âge : l'exemple des «Quatre Villes de la Côte »
}

Research on the trade circuits of northern Spain at the end of the Middle Ages: the example of the "four coastal cities"

Javier Añíbarro Rodríguez

\section{(2) OpenEdition}

\section{Journals}

\section{Édition électronique}

URL : http://journals.openedition.org/abpo/2399

DOI : $10.4000 / a b p o .2399$

ISBN : 978-2-7535-2129-2

ISSN : 2108-6443

\section{Éditeur}

Presses universitaires de Rennes

\section{Édition imprimée}

Date de publication : 30 juin 2012

Pagination : 125-143

ISBN : 978-2-7535-2127-8

ISSN : 0399-0826

Référence électronique

Javier Añíbarro Rodríguez, «Recherches sur les circuits commerciaux du nord de l'Espagne à la fin du Moyen Âge : l'exemple des "Quatre Villes de la Côte »", Annales de Bretagne et des Pays de l'Ouest [En ligne], 119-2 | 2012, mis en ligne le 30 juin 2014, consulté le 01 mai 2019. URL : http:// journals.openedition.org/abpo/2399; DOI : 10.4000/abpo.2399 


\title{
Recherches sur les circuits commerciaux du Nord de l'Espagne à la fin du Moyen Âge : l'exemple des «Quatre Villes de la Côte"
}

\author{
Javier AÑíBARRo RODRÍGUEZ \\ Profesor ayudante d'Histoire médiévale, \\ Université de Cantabrie - Groupe de recherche HISTAR
}

La présente étude se propose d'analyser les circuits commerciaux développés à la fin du Moyen Âge par quatre villes portuaires de la côte septentrionale de la péninsule Ibérique ${ }^{1}$ : Castro Urdiales, Laredo, Santander et San Vicente de la Barquera ${ }^{2}$. Connues par le passé sous l'appellation de "Quatre Villes de la Côte de la Mer " (Cuatro Villas de la Costa de la Mar), elles sont situées dans l'actuelle Communauté de Cantabrie, bordée à l'est par la Biscaye, à l'ouest par les Asturies, au sud par la province de Burgos et au nord par l'Atlantique, plus précisément par le golfe de Gascogne (golfo de Vizcaya). Il s'agit de quatre villes neuves fondées à la fin du XII ${ }^{\mathrm{e}}$ et au début du XIII ${ }^{\text {e }}$ siècle par Alphonse VIII de Castille qui concéda une charte de privilèges (fuero) à Castro Urdiales en 1163, à Santander en 1189, à Laredo en 1200 et à San Vicente de la Barquera en $1210^{3}$. Toutefois, au regard des

1. Cette étude s'inscrit dans le cadre du projet de recherche $\mathrm{I}+\mathrm{D}$ « Ciudades y villas portuarias en la articulación del litoral atlántico en la Edad Media ", financé par le Ministerio de Ciencia e Innovación, Gobierno de España (HAR 2009-08474), dirigé par Jesús Ángel Solórzano Telechea.

2. Seules sont prises en compte dans cette étude les quatre agglomérations portuaires ayant juridiquement le rang de ville (villa). De petites localités littorales servaient aussi de points de chargement et de déchargement des marchandises, à l'exemple de Santoña ou de San Martín de la Arena, droit que leur contestaient énergiquement les quatre villes.

3. Sur la côte cantabrique, Alphonse viII fonda également Fontarabie en 1203, Guetaria en 1209 et Motrico en 1209. Voir ARízAgA BolumBuru, Beatriz et BochacA, Michel, "Caractères généraux des villes portuaires du littoral castillan nord-péninsulaire au Moyen Âge ", dans Ports maritimes et ports fluviaux au Moyen Âge, Actes du Xxxve congrès de la Société des Historiens Médiévistes de l'Enseignement Supérieur Public, La Rochelle (2004), Paris, 2005, p. 63-78; SolóRZANo TelEcheA, Jesús Ángel, " Medieval Seaports of the Atlantic Coast of Spain ", International Journal of Maritime History, XXI, $n^{\circ} 1$ (June 2009), p. $81-100$. 
autres provinces de la côte septentrionale de la Castille, la Galice et les Asturies à l'ouest, la Biscaye et le Guipúzcoa à l'est, la côte de la Cantabrie était la moins densément urbanisée à la fin du Moyen Âge.

\section{Figure 1 - L'urbanisation de la côte septentrionale de la Castille à la fin du XIV siècle}

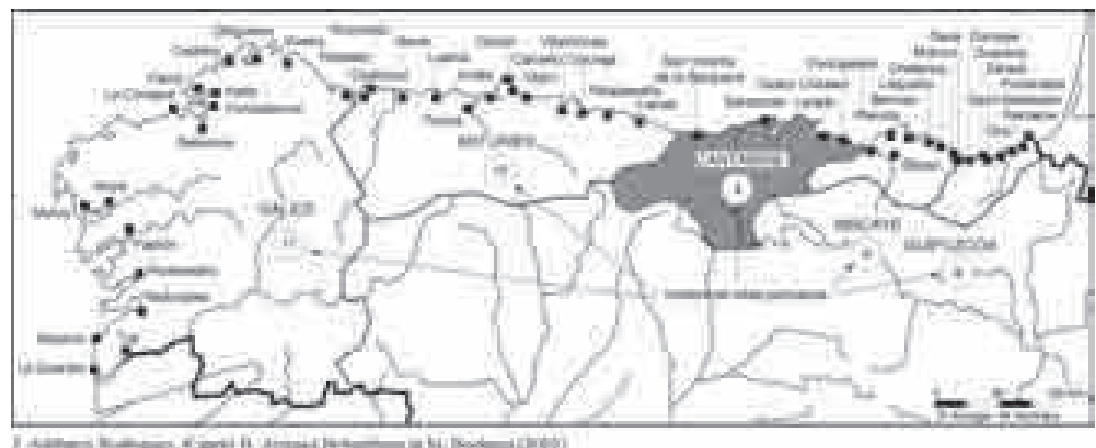

Leur développement jusque dans un XIV ${ }^{\mathrm{e}}$ siècle largement avancé est mal connu faute de sources. Il s'agit de petites villes par la superficie ${ }^{4}$. L'espace intra muros le plus étendu atteint péniblement 6 hectares dans le cas de Santander. Ces villes sont également petites par leur population. En l'absence de données fiscales concernant le nombre de feux, des ordres de grandeur approximatifs et difficiles à vérifier ont été avancés pour la fin du $\mathrm{XV}^{\mathrm{e}}$ siècle : autour de 1500 habitants à Santander, après que la ville ait été désolée par deux récurrences pesteuses en 1494 et surtout en 1497; 2200 à Castro Urdiales; 2800 à San Vicente de la Barquera; 3500 à Laredo ${ }^{5}$. Les gens de mer (pêcheurs et marins) représentaient une part importante de la population à côté des marchands. De ce fait, les activités en relation avec la mer (pêche, transport et commerce maritimes) occupaient une place importante dans leur économie. En vertu des franchises reçues lors de leur fondation, elles disposaient d'une large autonomie municipale et s'administraient elles-mêmes sous la direction d'un conseil de ville (concejo). Jusque dans la seconde moitié du $\mathrm{XV}^{\mathrm{e}}$ siècle, le pouvoir resta en grande partie aux mains des élites nobiliaires, reléguant le commun au second plan ${ }^{6}$.

4. Arízaga Bolumburu, Beatriz, "Villas Cántabras : permanencias urbanas ", Memoria histórica de Cantabria, Santander, 1996, p. 71-83.

5. Solórzano Telechea, Jesús Ángel, Santander en la Edad Media. Patrimonio, parentesco $y$ poder, Santander, 2002, p. 44.

6. SolóRZANO TElECHEA, Jesús Ángel, « Linaje, comunidad y poder : desarrollo y consolidación de identidades urbanas contrapuestas en la Castilla Bajomedieval ", Aragón el la Edad Media, Zaragoza, 2007, p. 71-93. 
Figure 2 - Plans des Quatre Villes de la Côte (J. Añibarro Rodriguez, d'après B. Arizaga Bolumburu [1996D
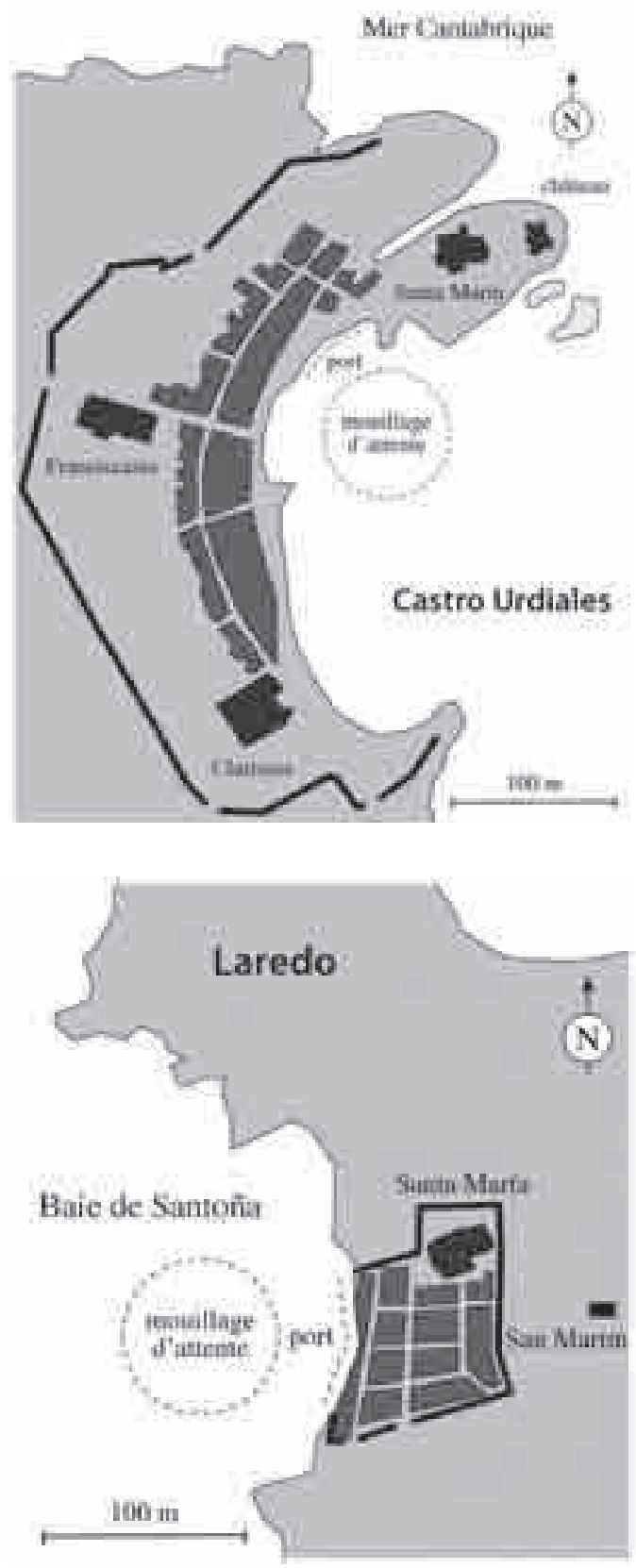

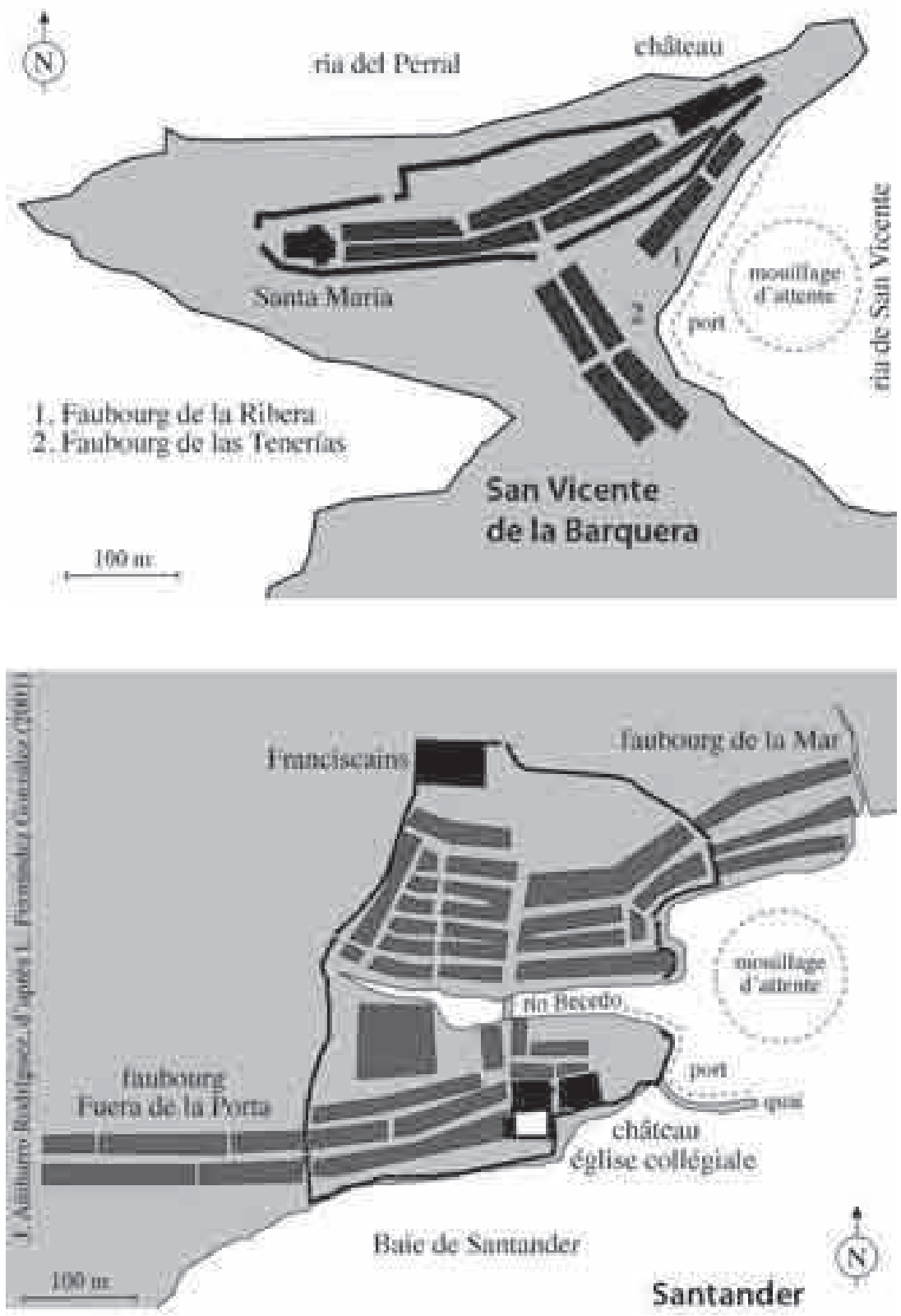

Du point de vue des infrastructures portuaires, Santander comptait une nette avance sur les trois autres ports cantabres du fait de la présence des atarazanas (chantier naval royal similaire au clos des galées du roi de 
France), connues dès 1396, et d'un quai de pierre attesté en 1434. À Laredo, Castro Urdiales et San Vicente, la construction de quais ne débuta pas avant le milieu du $\mathrm{XV}^{\mathrm{e}}$ siècle, les activités portuaires se faisant à même la grève connue sous le nom de la Ribera ${ }^{7}$. Comme dans de nombreux autres ports de la façade de l'Europe atlantique, la projection maritime à la fin du Moyen Âge se faisait à partir d'infrastructures portuaires rudimentaires mais qui suffisaient au regard des moyens techniques limités de l'époque ${ }^{8}$.

À travers l'étude des circuits commerciaux tels qu'ils se présentaient à la fin du XV et au début du XVI ${ }^{\mathrm{e}}$ siècle nous tâcherons d'apprécier la place de ces quatre ports dans les trafics régionaux. Comme les autres ports de la côte septentrionale, les Quatre Villes de la Côte jouèrent le rôle d'interfaces d'échange (gateway) avec l'intérieur de la Castille en servant de porte d'entrée et de sortie des produits ${ }^{9}$. Il reste cependant à évaluer quelle fut leur place exacte au sein de la hiérarchie portuaire nord péninsulaire et qui, des marchands locaux ou de ceux venus de l'extérieur, furent les acteurs les plus actifs, enfin comment s'établissaient les relations avec l'hinterland. Pour les besoins de cette étude nous avons mobilisé les sources inédites disponibles aux Archives Générales de la Couronne de Castille (Simancas) et à la Chancellerie royale à Valladolid. Il s'agit essentiellement de pièces judiciaires (procès) d'une part, et requêtes des conseils urbains aux souverains de Castille, d'autre part. Les autres documents, en partie publiés, se trouvent aujourd'hui dans les archives provinciales et municipales de Cantabrie et de Biscaye ${ }^{10}$. Ce sont des comptes et des documents fiscaux du conseil de ville (Castro Urdiales, Lekeitio), des recueils d'ordonnances (Laredo, Castro Urdiales, Bilbao) et de registres des délibérations municipales (Bilbao), et de documents judiciaires (Laredo). Ces documents éclairent notre sujet de façon partielle et fragmentaire à partir de cas particuliers et isolés. Toutefois ces données éparses mettent en lumière des exemples concrets qui reflètent une situation plus générale. Au-delà de leur caractère qui peut paraître anecdotique au premier abord, ils renvoient à des réalités historiques de portée plus générale. En l'absence de registres de douane, de comptes de marchands ou de confréries de pêcheurs, aucune approche quantitative n'est possible. Ce type de démarche quelque peu " impressionniste " est cependant le lot habituel de la recherche en histoire médiévale.

7. SolóRZANo Telechea, Jesús Ángel y AÑIBARRo RodRigueZ, Javier, « Infraestructuras e instalaciones portuarias fluviales e hídricas en las villas del Norte peninsular a finales de la Edad Media : las obras públicas como instrumentos del poder ", in María Isabel del Val Validivieso y Olatz Villanueva Zubizarreta (eds.), Musulmanes y Cristianos frente al Agua en las Ciudades Medievales, Santander, 2008, p. 275-305.

8. BOCHACA, Michel et SARRAZIN, Jean-Luc (dir.), Ports et littoraux de l'Europe atlantique. Transformations naturelles et aménagements humains (XIV -XVI siècles), Rennes, PUR, 2007.

9. ORVIETANI BusCh, Silvia, «Mediterranean ports : interdisciplinary keys to their understanding ", Medieval Mediterranean Ports. The Catalan and Tuscan Coasts, 1100 to 1235, Leiden, 2001, p. 255- 265.

10. AÑ́íBARRo RodrígueZ, Javier, « Las redes portuarias en las Cuatro Villas de la Costa de la Mar durante la baja Edad Media : avances de su estudio ", Redes sociales y económicas en el mundo bajomedieval, Valladolid, 2011, p. 177-196. 


\section{Une géographie portuaire et des activités économiques fortement contraintes par les données naturelles}

San Vicente de la Barquera, Santander, Laredo et Castro Urdiales s'égrènent d'ouest en est le long des 284 kilomètres de côtes de la Cantabrie, caractérisées par de hautes falaises de calcaire ou de grés tombant en à pic dans l'Atlantique. Vue de la mer, la côte se présente comme un mur abrupt qui laisse cependant place de point en point au débouché de petits fleuves côtiers ${ }^{11}$, à l'exemple de l'Escudo et du Gandarilla à l'embouchure conjointe desquels San Vicente de la Barquera fut édifiée. Par endroits, le tracé découpé de la côte ménage des conches comme celles de Castro Urdiales et de Laredo, voire une vaste baie dans le cas de Santander. Ces échancrures abritées des houles et des vents venus de l'océan ont fixé les principaux ports. Elles offrent des conditions naturelles favorables pour l'établissement de noyaux de population et le développement d'activités maritimes. Presque toutes disposent d'une échine montagneuse qui les protège au nord, offrant un site défensif et abritant les navires des vents du nord et d'ouest. L'approvisionnement en eau douce y est facile. La position à l'embouchure d'un fleuve côtier ou à proximité de celle-ci facilite les communications avec l'intérieur, même si la proximité de hauts massifs montagneux limite considérablement l'extension du réseau hydrographique. Cette situation prend toute sa valeur pour l'acheminement vers la côte des productions de l'arrière-pays, entre autres minérales. Il existait d'autres cales et lieux de chargement et de déchargement qui pouvaient servir de ports secondaires, mais les autorités municipales des Quatre Villes veillèrent à faire respecter le monopole commercial dont leur port jouissait, évitant ainsi le développement de ports secondaires concurrents ${ }^{12}$.

Toutefois vers l'intérieur, à une quinzaine de kilomètres en arrière de la côte, le relief devient vigoureux dès lors qu'on aborde les monts cantabriques. Dépassant les 500 mètres d'altitude, cette chaîne montagneuse forme une puissante barrière d'une centaine de kilomètres de large en moyenne et dont les plus hauts sommets dépassent les 2000 mètres. Les communications avec l'intérieur de la Castille, en direction des provinces de Burgos et de Palencia, étant difficiles, les habitants des villes littorales se sont très tôt tournés vers la mer pour en tirer les moyens de leur subsistance et pour rompre leur relatif isolement.

11. ORTEga VAlCÁRCEL, José, "Cantabria ", dans Geografía de España, vol. IV, Barcelona 1990, p. 461-584, p. 461-468.

12. Deux exemples sont fournis par la volonté des habitants de San Vicente de la Barquera de détruire le port qui se construisait à Comillas en 1480 (SOLÓRZANO TELECHEA, Jesús Ángel, Catálogo del patrimonio documental de Cantabria, Santander, 1999, doc. 37, p. 22) et par la lutte, à partir de 1434, de Santander contre le Marquis de Santillana pour le contrôle du port de San Martín de la Arena (SOLóRZANo TELECHEA, Jesús Ángel, FERnÁNDEZ GONZÁlEz, Lorena, Conflictos jurisdiccionales entre la villa de Santander y el Marquesado de Santillana en el siglo XV, Santander, 1996, doc. 2, p. 63-249). 
Du point de vue de l'agriculture San Vicente de la Barquera, Santander, Laredo et Castro Urdiales souffraient d'un lourd handicap lié à la faible production locale de céréales, en particulier de froment, à une époque où celui-ci constituait la base de l'alimentation. Les habitants de la région cultivaient d'autres céréales panifiables comme le mil et le seigle, mais la production céréalière fut toujours limitée du fait du relief montagneux et du climat humide. Sols et climat se prêtaient un peu mieux à la culture de la vigne sur quelques terroirs autour des villes, comme le montrent les déclarations recueillies lors d'une enquête menée en 1516 par le procureur de la municipalité de San Vicente de la Barquera. Les habitants déclarèrent qu'en “las tierras tenyan pocas lavores salvo solo algunas vyñas ${ }^{13}$ ». Le vin produit était de mauvaise qualité mais il suffisait à satisfaire la demande locale et quelques surplus étaient exportés à l'occasion vers l'Irlande et l'Angleterre $^{14}$. Le manque chronique de céréales gênait le développement de ces populations qui souffrirent de fréquentes crises de subsistance.

Compte tenu des contraintes naturelles (relief montagneux et manque de terres cultivables), l'espace exploitable par les habitants des Quatre Villes était exigu. À part l'élevage ${ }^{15}$, la population se tourna vers la mer pour trouver de quoi se nourrir. La pêche occupait une place prépondérante dans l'économie et la société des Quatre Villes à la fin du Xv ${ }^{\mathrm{e}}$ siècle ${ }^{16}$. Le poisson représentait une ressource importante pour l'alimentation directe des habitants et comme produit commercialisable une fois séché et salé. Juan de Oreña, habitant de San Vicente de la Barquera, affirmait en 1516 que plus des neuf dixièmes de la population de la ville dépendaient directement ou indirectement de la mer : « que casy todos ellos viben por el ofiçio

13. Blanco Campos, Emma, Álvarez Llopis, Elisa, García de Cortázar, José Ángel, Documentación referente a Cantabria en el Archivo General de Simancas. Sección Cámara de Castilla (Años 1483-1530), Santander, 2005, doc. 589, p. 370; Archivo General de Simancas, Memoriales, Leg. 120, doc. 80, $\mathrm{f}^{\circ} 6$ (désormais : AGS). En 1504, San Vicente de la Barquera n'aurait disposé que de 40 fanegas de blé pour alimenter 10000 personnes. Au-delà de l'exagération manifeste, la pénurie frumentaire était un fait récurrent et un sujet de préoccupation constant. Voir Blanco CAMPos, Emma, ÁlVAREZ LLOPIS, Elisa, GarCía DE CORTÁZAR, José Ángel, Documentación referente a Cantabria, op. cit., doc. 163, p. 123.

14. Solórzano Telechea, Jesús Ángel, Colección Diplomática del Archivo Municipal de Santander. Documentación Medieval (1295-1504). Santander 1995, doc. 136, p. 192; BLANCO CAMPOS, emma, álVAREZ lLOPIS, elisa, gARCíA DE CORTÁZAR, José Ángel, Libro del concejo (14941522) y documentos del archivo municipal de Castro Urdiales, Santander, 1996, doc. 15, p. 133; Baró PAZos, Juan, Galván RIVERo, Carmen, Libro de Ordenanzas de la villa de Castro Urdiales (1519-1572). Santander, 2006, p. 86.

15. Sur le rôle de l'élevage, voir AÑíBARRO RODRíGuEZ, Javier, La implantación urbana medieval en la Costa de Cantabria. ¿Creación original o herencia del pasado?, Santander, 2010, p. 131 et 153.

16. L'importance de la pêche se mesure à travers les taxes (alcabalas) grevant la commercialisation du poisson. En 1502, elles représentaient $58 \%$ des recettes de l'ensemble des alcabalas de San Vicente, 51 \% à Castro Urdiales, 50 \% en Laredo et $43 \%$ à Santander. Voir Solinís Estallo, Miguel Ángel, La alcabala del rey, 1474-1504, Santander, 2003, p. 166167, tableau VIII ; ARízAGA BoLUMBURU, Beatriz, " Las actividades económicas de las villas marítimas del norte peninsular ", Las sociedades urbanas en la España Medieval, XxIX Semana de estudios medievales, Pamplona, 2003, p. 215. 
de la mar en ella, e no tienen otro ofiçio esçebto algunos. Muy pocos usan de ofiçios de ser letrados, escribanos, sastres, barberos e ferreros e otros ofiçios de manera que casy de treinta partes, las veynte e nueve vyven por el oficio e trabto de la mar $^{17}$ ". Il était possible de pêcher tout le long de la côte. La plateforme continentale, réduite à 10 kilomètres devant Laredo, s'étendait sur 20 kilomètres face à Santander et sur 25 à hauteur de Castro Urdiales; bien qu'étroite, elle était riche en poissons. Au-delà du talus continental (-200 mètres), les fonds plongeaient rapidement jusque vers les grandes fosses marines (-2000 mètres) empêchant toute pratique de la pêche, que ce soit avec des lignes ou des filets ${ }^{18}$. À partir de la fin du XIV siècle, certains pêcheurs tentèrent leur chance en allant pêcher au loin. Combinant la pêche proprement dite avec la conservation du poisson et sa commercialisation, ils partaient pour de longues campagnes pouvant durer cinq ans et plus ${ }^{19}$. Ils se rendaient en Galice où abondaient les sardines, sur la Côte de Berbérie, située entre les îles Canaries et la côte d'Afrique, enfin en Irlande ${ }^{20}$. Le poisson capturé lors de ces campagnes lointaines était salé au fur et à mesure à bord des navires ${ }^{21}$. Il fallait pour cela embarquer du sel qui venait des grands centres de production européens : Aveiro et Setubal au Portugal, Aunis et Saintonge via La Rochelle et la baie de Bourgneuf en France. Ce sel acheté principalement dans les ports de Galice et des Asturies faisait l'objet d'un commerce de redistribution à destination de la Cantabrie ${ }^{22}$. À partir du XVI ${ }^{\mathrm{e}}$ siècle le sel d'Andalousie en provenance de Séville fit son apparition sur la côte cantabrique.

17. Blanco Campos, Emma, Álvarez llopis, Elisa, García de Cortázar, José Ángel, Documentación referente a Cantabria..., op. cit., doc. 589, p. 370; AGS, Memoriales, Leg. 120 , doc. $80, \mathrm{f}^{\circ} 5$.

18. Ortega Valcárcel, J., Gentes de Mar en Cantabria. Santander, 1996. p. 39.

19. AGS, Cámara de Castilla, Pueblos, leg. 17, Agosto 18, 1514.

20. Solórzano Telechea, Jesús Ángel, VÁzquez Álvarez, Roberto, Arízaga Bolumburu, Beatriz, San Vicente de la Barquera en la Edad Media : una villa en conflicto. Archivo de la Real Audiencia y Chancillería de Valladolid (1241-1500), Santander, 2004, doc. 12, p. 76 et doc. 40, p. 309 : les pêcheurs de San Vicente allaient pêcher en groupe dans " la mar de Berbería " et un seul navire pouvait charger jusqu'à " 235 douzaines de poisson "; doc. 46, p. 367, la Berbérie et l'Irlande sont désignées comme les principaux endroits où se pratiquait la pêche hauturière. SAINz DíAz, Valentín, Notas históricas sobre la villa de San Vicente de la Barquera, Santander 1986, p. 529.

21. Sur le processus de conservation du poisson, voir FERREIRA PRIEGUE, Elisa, Galicia en el comercio marítimo medieval, La Coruña, 1988, p. 146-147; GONZÁLEZ ECHEGARAY, Joaquín, Manual de Etnografía Cántabra, Santander, 1988, p. 158; ARízAGA BolumBuru, Beatriz, « Las actividades económicas... ", op. cit., p. 213.

22. Ruiz De LA PeÑA, Juan Ignacio, Las “ polas » asturianas en la Edad Media. Oviedo 1981, p. 233; URIA Riu, Juan, Estudios sobre la Baja Edad Media asturiana, Oviedo, 1979, p. 326332 ; FERREIRA PRIEGUE, Elisa, Galicia en el comercio marítimo medieval, La Coruña, 1988, p. 156-161; SolóRZANo TelecheA, Jesús Ángel, Colección documental de la Villa medieval de Santander en el Archivo General de Simancas (1326-1498), Santander, 1999, doc. 61, p. 111. Les importations de sel en provenance de la Biscaye semblent plus rares : SOLÓRZANO TelecheA, Jesús Ángel, Colección diplomática..., op. cit., doc. 248, p. 381. 
Figure 3 - La projection atlantique des quatre villes de La côte à la fin du Moyen Âge

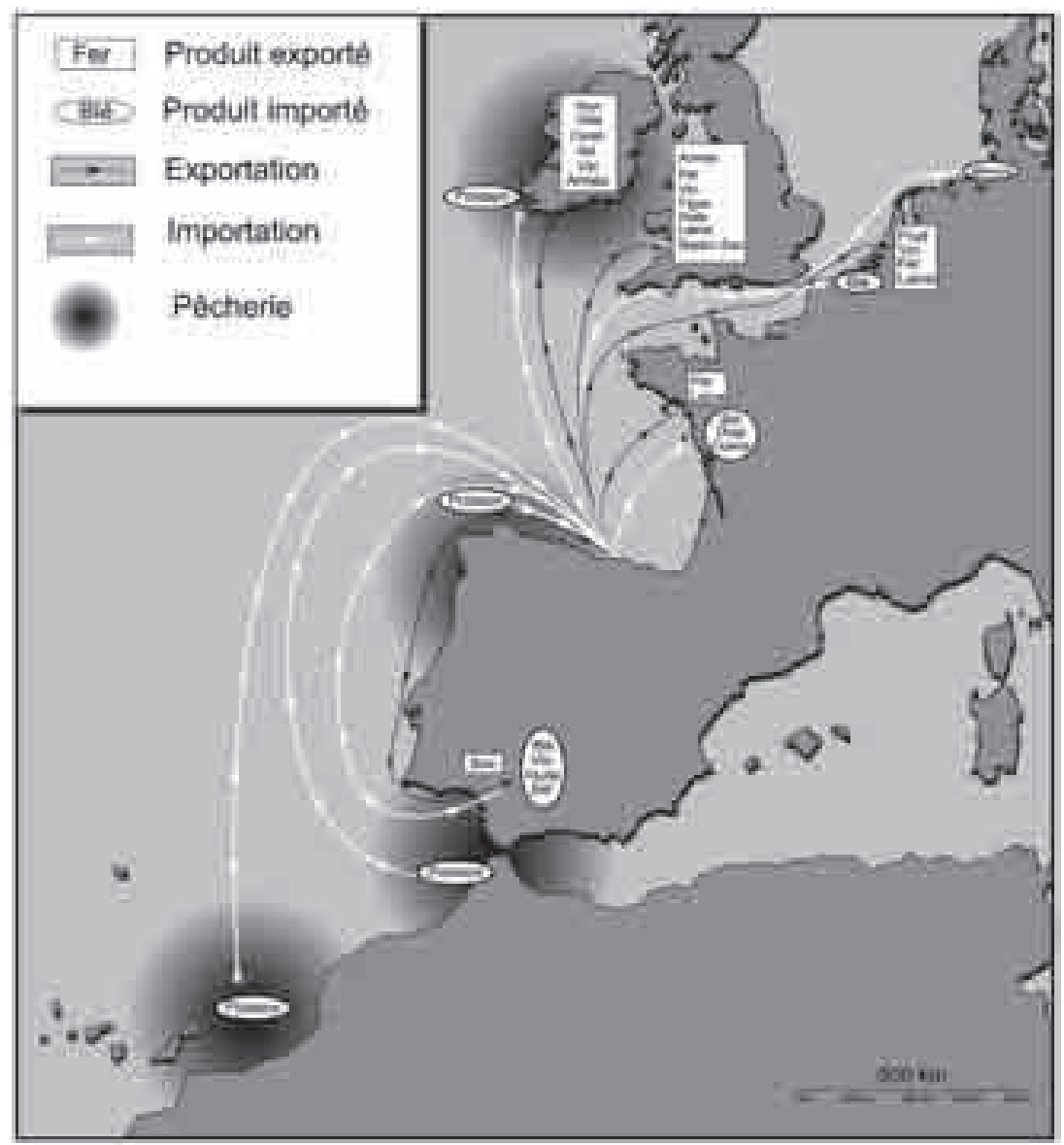

La Cantabrie disposait toutefois de deux ressources naturelles qui permettaient des produits d'expotation, le fer et le bois. Le fer provenait du centre de la Cantabrie et surtout de la partie orientale aux confins de la Biscaye $^{23}$. Il existait deux gisements d'une certaine importance, l'un à l'est dans la vallée de Guriezo, formant le prolongement du gisement biscayen de Somorrostro, l'autre au centre de la province au sud de Santander, à Cabárceno. Le minerai était extrait à partir de veines; il était ensuite transformé dans des fourneaux situés à 10 ou 15 kilomètres dans l'arrière-pays, puis acheminé jusqu'à la côte au moyen de chaloupes qui empruntaient

23. SolóRzano Telechea, Jesús Ángel, "La producción y comercialización del Hierro ", Studi Medievali, 3aㅗㄹ seire, anno XLVII, fasc. I, Giugno 2006, p. 71-109. 
les quelques voies d'eau disponibles. Région montagneuse abondamment couverte de forêts, la Cantabrie disposait d'importantes ressources en bois. À côté du chêne, très apprécié pour la fabrication des navires, on utilisait aussi le hêtre, le châtaignier et d'autres espèces. Transformé en charbon, le bois alimentait les fourneaux des ferrons, permettant la réduction du minerai de fer grâce à son pouvoir calorifique élevé ${ }^{24}$. Cette utilisation massive amena par exemple la municipalité de Castro Urdiales à réglementer la coupe des bois communaux par les habitants de la ville ${ }^{25}$.

\section{L'articulation commerciale entre les ports de la côte et l'intérieur de la Castille}

En l'absence d'un réseau hydrographique développé et du fait de la présence d'une barrière montagneuse parallèle à la côte, les échanges avec l'intérieur de la Castille se faisaient essentiellement par voie terrestre. La circulation était lente car il fallait franchir les monts cantabriques, raison pour laquelle les marchands employaient de préférence des mulets pour le transport des marchandises. En 1494, on voit un groupe de muletiers transportant du sel de Santander à la Tierra de Campos former une caravane de 16 mules $^{26}$. Tout au long du chemin, marchands et muletiers pouvaient faire étape dans les nombreuses villes qui jalonnaient leur itinéraire. À partir de San Vicente de la Barquera, la route traversait Cabezón de la Sal, Cabuérniga, Los Tojos, Espinilla, Aguilar de Campoo, Herrera de Pisuerga et Carrión de los Condes. De là ils pouvaient gagner Burgos ou Palencia. Depuis Santander, hommes et bêtes passaient par Santillana puis suivaient la vallée du Besaya vers le sud jusqu'à Reinosa. Deux options s'offraient alors à eux : rattraper la route venant de San Vicente à hauteur d'Espinilla ou bien se diriger directement vers Burgos ${ }^{27}$. Sur ces routes principales venaient se greffer des chemins secondaires qui innervaient les différentes vallées de la province ${ }^{28}$. L'ensemble formait un réseau dense, surtout dans la partie occidentale de la Cantabrie.

24. Arízaga Bolumburu, Beatriz, "Las actividades económicas... ", op. cit., p. 195-200.

25. Baró Pazos, Juan, Galván Rivero, Carmen, Libro de Ordenanzas..., op. cit., p. 111, $\mathrm{f}^{\circ} 20$ $\mathrm{v}^{\circ}$; concernant les chênes et d'autres espèces d'arbres, ibid., p. 172, $\mathrm{f}^{\circ} 61 \mathrm{r}^{\circ}$.

26. La vente du sel par les Quatre Villes est documentée par un procès qui opposa en 1504 Salinas de Añana à Santander et à San Vicente de la Barquera Voir SolóRZANo TelecheA, Jesús Ángel, Los Conflictos del Santander medieval, Santander, 1999, doc. 88, p. 452. La manière dont les muletiers travaillaient est dévoilée à l'occasion d'un conflit survenu avec des habitants de Herrera qui dérobèrent "catorce asémilas e dos roçines cargados de sal con sus aparejos a tres atyjareros que lo llevavan [...] a la dicha villa de Herrera " (voir Solórzano TelecheA, Jesús Ángel, Colección documental..., op. cit., doc. 88, p. 150-151).

27. MolÉNAT, Jean-Pierre, "Chemins et ponts du nord de la Castille au temps des Rois Catholiques ", Mélanges de la Casa de Velásquez, n 7, 1971, p. 115-162.

28. Pour plus de détails sur les chemins médiévaux, voir FERnÁndEz GonZÁLEz, Lorena, Santander. Una ciudad medieval, Santander, 1996, p. 121-153 et ÁlVAREz LloPIS, Elisa, BlaNCO CAmpos, Emma, " Las vías de Comunicación en Cantabria en la Edad Media ", I Encuentro de Historia de Cantabria. Santander, 1999, p. 491-522. 
Les circuits commerciaux du Nord de l'Espagne à la fin du Moyen Âge

Figure 4 - Routes terrestres et circulation des produits entre la côte cantabrique et l'intérieur de la Castille à la fin du Moyen Âge

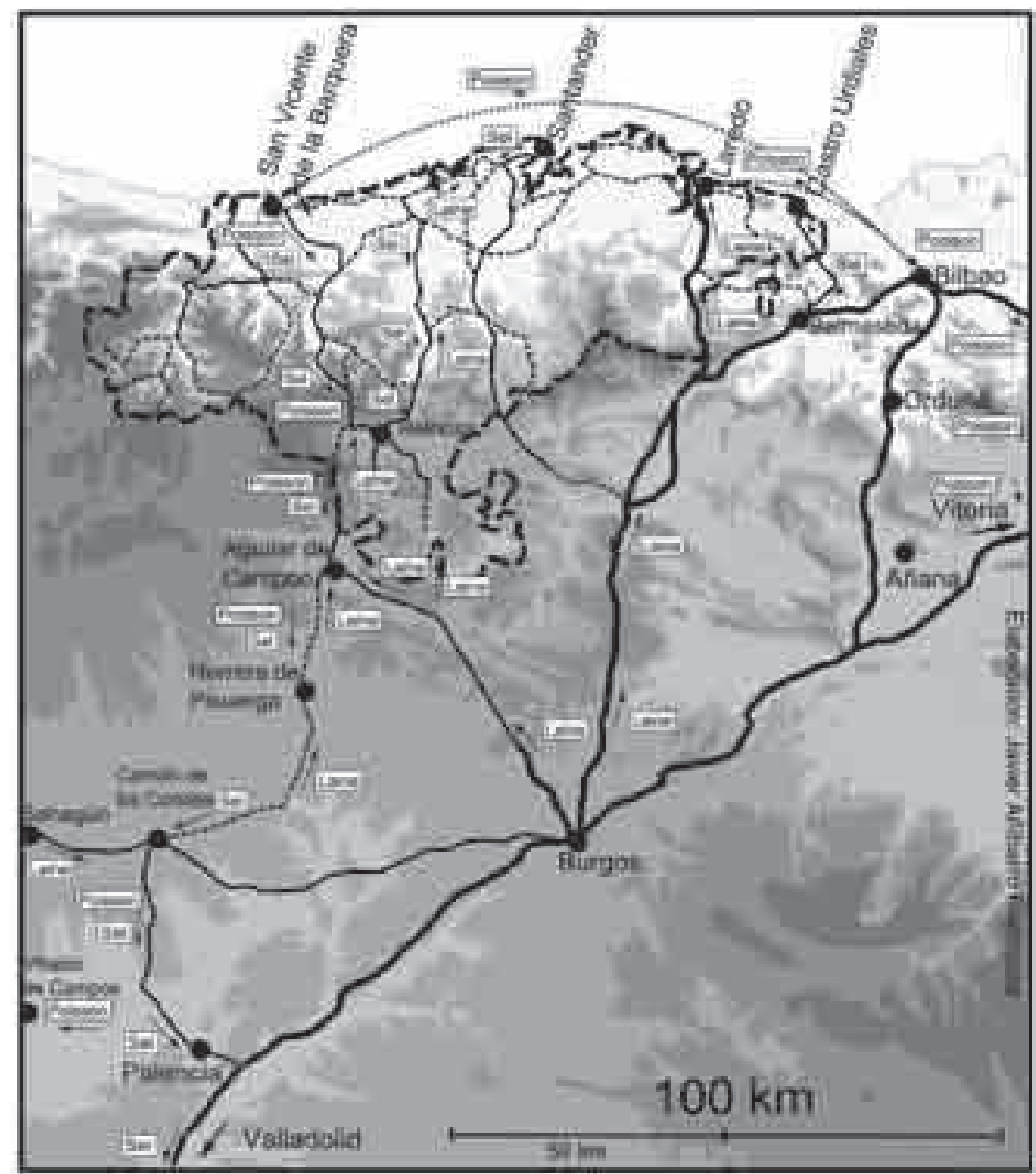

Chemin important

Chamin de troistrme ordre

Chemin de second orde' Sel Marchandise

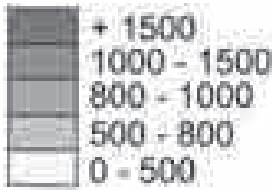


Les produits acheminés vers l'intérieur étaient, entre autres, le fer, le sel et le poisson. En 1401, Fernando Sánchez, habitant de Villasevil de Toranzo, promit de livrer en deux fois à Fernando Gutiérrez de Escalante, de Santander, 51 douzaines de pièces de fer semi-ouvré. Les intérêts perçus pour cette transaction lui valurent des démêlés avec la justice, grâce auxquels elle nous est connue ${ }^{29}$. Certains marchands de la côte réussirent à mettre la main sur le trafic du sel à destination de la Tierra de Campos, région située de l'autre côté des monts cantabriques, battant en brèche le monopole de Salinas de Añana. Toutefois les Quatre Villes disposaient de privilèges royaux qui leur permettaient de s'affranchir de ce monopole ${ }^{30}$.

Grâce au procédé de conservation par séchage et salaison, les marchands des Quatre Villes purent distribuer le poisson dans un large rayon. En février 1489, deux marchands de San Vicente de la Barquera, Juan de Oreña et Pero Álvares Barvero, entreposèrent à Villalón plus de 50 milliers de sardines séchées et salées. Ils devaient escompter en tirer un bon prix car la location du lieu de stockage s'élevait à 4400 maravédis; et encore, ils s'étaient entendus pour partager les frais. Le poisson parvenait aussi jusqu'à Aguilar de Campoo, Orduña et d'autres secteurs du nord de la Castille $^{31}$. En 1488, des habitants de San Vicente s'engagèrent à livrer à Aguilar des congres et d'autres poissons pour une valeur de 5000 maravedis $^{32}$. Au début du Xve siècle, d'après le témoignage de l'archiprêtre Hita, les saumons de Castro Urdiales étaient renommés ainsi que les langoustes de Santander, les dorades (besugos) et les harengs de Bermeo et le congre de Laredo $^{33}$. Le sábalo, sorte de grande alose, parvenait jusqu'au marché de Madrid si on en croit les tarifs des droits perçus ${ }^{34}$. Au début du XVI ${ }^{\mathrm{e}}$ siècle, les habitants de San Vicente de la Barquera écoulaient du poisson séché de l'autre côté de la Cordillère cantabrique, à Herrera de Pisuerga et à Orduña.

Tous les circuits commerciaux ne partaient pas directement de l'une des quatre villes portuaires cantabres. Elles disposaient de privilèges qui permettaient à leurs habitants de vendre leurs produits à partir d'autres villes. En 1505, des pêcheurs de San Vicente de la Barquera de retour d'une campagne de pêche en Irlande déchargèrent leurs prises à Bilbao où ils savaient pouvoir les y vendre. Les marchands devaient aussi compter avec l'hostilité de certains seigneurs, en particulier ceux d'Herrera de Pisuerga,

29. Archivo Histórico Nacional, Osuna, leg. 1785-1. Voir aussi SolóRZANo TelECHEA, Jesús Ángel, " La producción y comercialización del Hierro... ", op. cit., p. 83-84. L'interdiction de l'usure édictée par Alphonse x fut rappelée lors des Cortes d'Alcalá en 1386.

30. Solórzano TelecheA, Jesús Ángel. Patrimonio documental de Santander en los Archivos de Cantabria. Documentación medieval (1253-1515), Santander 1998, doc. 69, p. 148.

31. Solórzano Telechea, Jesús Ángel, Vázquez Álvarez, Roberto, Arízaga Bolumburu, Beatriz, San Vicente de la Barquera..., op. cit., doc. 20, p. 128.

32. Ibidem, doc. 18, p. 113 et doc. 20, p. 128.

33. GarCía De CoRTÁZAR, José Ángel, Vizcaya en el siglo XV. Aspectos económicos y sociales, Bilbao, 1966, p. 113.

34. Puñal Fernández, Tomás, El Mercado en Madrid en la Baja Edad Media, Madrid, 1992, p. 172 . 
dans la province de Palencia, et ceux d'Orduña, une enclave biscayenne dans le territoire de la province d'Alava, qui voyaient d'un mauvais œil les franchises royales dont ils jouissaient et qui continuaient à exiger le paiement des taxes (diezmo) et n'hésitaient pas à faire saisir leurs marchandises $^{35}$.

\section{Des ports servant de débouchés aux produits de l'intérieur mais incapables de résister à la concurrence de Bilbao}

Les ports des Quatre Villes servaient aussi de sortie à la laine castillane qui partait de Burgos à destination de la Flandre et de l'Angleterre. Mais les deux routes principales évitaient autant que possible les monts cantabriques. L'une, à l'est, passait par Briviesca, Pancorbo, Orduña et Bilbao; l'autre passait par Miranda de Ebro et Vitoria, et de là se divisait en deux branches, l'une conduisant à Durango et Bilbao, l'autre à Saint-Sébastien par Segura et Tolosa. Le chemin le plus court était sans conteste celui reliant Burgos à Laredo, mais il traversait la cordillère cantabrique par le col de Los Tornos. En mauvais état et d'un coût d'entretien élevé, cette voie fut délaissée au profit des deux autres itinéraires, surtout celui qui aboutissait à Bilbao ${ }^{36}$. Les routes de Burgos à San Vicente de la Barquera et à Santander, qui devaient franchir des secteurs élevés et enneigés en hiver, jouaient un rôle secondaire. Les deux villes portuaires cantabres qui parvinrent à capter une partie des trafics de Santander ${ }^{37}$ et Laredo ${ }^{38}$. Toutefois, d'un accès plus facile en venant de Burgos, Bilbao s'imposa comme le principal port de sortie des laines castillanes et consolida ainsi son ascendant sur les ports voisins de la Cantabrie.

35. AGS. Cámara de Castilla. Pueblos, leg. 17, doc. $334, \mathrm{f}^{\circ} 1 \mathrm{v}^{\circ}$, « en pasando el dicho pescado por la çibdad de Ordeña, diz que çiertos dismeros e fiadores vuestros prendieron el dicho pescado e le llevaron el dismo de ello contra su voluntad, que serían hasta ocho myll maravedíes [...] e les dio información como era vesinos de la dicha villa de San Viçente, naturales de ella. Diz que non la quesyeron obedeçer ny cumplir... ". Ibid., $\mathrm{f}^{\circ} 2 \mathrm{v}^{\circ}$. Le même problème est attesté à en Herrera de Pisuerga : "se tomaron çiertas cargas de pescado descaminado a los de la villa de Sant Viçente de la Barquera e que por otras cartas yo mandé tornar el dicho pescado a sus dueños".

36. MolÉnAt, Jean-Pierre, "Chemins et ponts du Nord de la Castille... ", op. cit., p. 115162 ; Casado Alonso, Hilario, Señores, Mercaderes y Campesinos, Valladolid, 1987, p. 251260 ; PÉREz BustamANTE, Rogelio, "El marco jurídico para la construcción y reparación de caminos. Castilla siglos XIV y XV ", Les communications dans la péninsule ibérique au Moyen Âge, Paris, 1981, p. 163-178; Álvarez Llopis, Elisa y Blanco CAMPos, Emma, " Las vías de comunicación... ", op. cit., p. 491-521; FERNÁNDEZ GONZÁLEZ, Lorena, Santander, una ciudad medieval, Santander, 2001, p. 121-153.

37. Solórzano TelecheA, Jesús Ángel, Colección Diplomática..., op. cit., doc. 255, p. 404, $406,422,427$.

38. SuÁrez Fernández, Luis, Política internacional..., op. cit., t. II, 1482-1488, doc. 75 , p. 335. AGS, Registro General del Sello, Registros Normales, Sello, vol. v, $\mathrm{f}^{\circ} 23, \mathrm{n}^{\circ} 1650,13$ noviembre 1487. CASADO AlONSO, Hilario, "Comercio internacional y seguros marítimos en Burgos en la época de los Reyes Católicos ", Congreso Internacional Bartolomeu Dias e a sua época, t. III, Economía e Comercio marítimo, Porto, 1989, p. 585-608 (p. 605). 
Il exista un projet pour intensifier les échanges entre Burgos et Santander et faire de cette ville le principal centre portuaire de la côte nord de la Castille $^{39}$. Les avantages de la liaison Santander-Burgos y étaient mis en avant : "Los caminos de Santander son abiertos y mejores en forma que permiten el tránsito de carros, lo que no sucede desde Bilbao o Laredo, cuyos caminos no permiten más que el paso de caballerías por pie de mulo. " Toutefois, la route traversait des territoires sous contrôle du connétable de Castille; celui-ci levait des taxes sur la circulation des marchandises préjudiciables aux intérêts des marchands. Au milieu du Xve siècle, à la faveur du conflit opposant les marchands de Bilbao et de Burgos, Santander réussit à signer des accords commerciaux avec le consulat de Burgos qui présidait aux intérêts économiques des marchands de cette ville. Une partie des exportations de laines se détourna un temps vers son port mais Santander fut incapable de consolider cet avantage et de rivaliser durablement avec Bilbao, qui eut tôt fait de réaffirmer sa suprématie dès lors que les tensions avec Burgos s'apaisèrent ${ }^{40}$.

Les principales routes qui traversaient les monts cantabriques convergeaient vers Burgos. Les marchands castillans envoyaient depuis cette ville les ballots de laine vers Santander, Laredo et Bilbao pour les charger sur des navires à destination de l'Angleterre et de la Flandre. Toutefois, les quantités de laine transitant par ces divers ports n'étaient pas les mêmes. La plus grande partie était exportée par Bilbao et Laredo, tandis que la route de Santander, tout en disposant d'infrastructures correctes, était reléguée au second plan. Le recul fut marqué à partir de la fin du XV $\mathrm{V}^{\mathrm{e}}$ siècle après que Santander ait connu une épidémie de peste. À en croire des témoins, le déclin de Santander est consommé au début du $\mathrm{XvI}^{\mathrm{e}}$ siècle. En 1504 un habitant de la ville déclarait que " se acuerda avía çerca de myll vesinos en la dicha villa de Santander, asy de ruanos e mercaderes e mareantes y pescadores, e avía en ella grande trabto de lanas e fierro e maderas y pannos e otras mercaderías, e naos e carabelas ansy de la villa commo de fuera de ella, que lo lebaban a Flandes e a otras tierras las tales mercaderías, e agora con esta mortandad que ha tres o quatro annos que tanta es muerta mucha gente e non hae trabto ninguno en ella, salbo por todo perdido y non hae doscientos vesinos en la villa e alquerías ${ }^{41} "$.

La faible population des Quatre Villes, conséquence des crises de subsistance et des épidémies, limita leur rôle comme pôles de structuration de l'espace régional dans le nord de la Castille ${ }^{42}$. Leur développement fut

39. SolóRZAno TelecheA, Jesús Ángel, Patrimonio documental de Santander, op. cit., doc. 225, p. 283. El documento es una copia del siglo xx y carece de fecha. Il s'agit des dépositions des témoins présentés par Santander, dans un procès l'opposant au connétable de Castille. Ceux-ci mettent en avant l'avantage de faire passer les marchandises par Santander plutôt que par Laredo et Bilbao.

40. Solórzano TelecheA, Jesús Ángel, Patrimonio documental de Santander..., op. cit., doc. 53, p. 128; Id., "Santander y la construcción... ", op. cit., p. 53.

41. SólóRzano Telechea, Jesús Ángel, Colección diplomática..., op. cit., doc. 225, p. 404.

42. Prevenier, Walter, Sosson, Jean-Pierre, Boone, Marc, "Le réseau urbain en Flandre (XIII ${ }^{\mathrm{e}}-\mathrm{XIX}{ }^{\mathrm{e}}$ siècle) : composantes et dynamique ", Le réseau urbain en Belgique dans une perspective historique (1350-1850), Bruxelles, 1992, p. 157-200. 
aussi fortement contraint par leur relatif isolement au nord de la cordillère cantabrique par rapport aux centres économiques moteurs de la Castille comme Burgos et Medina del Campo. À défaut d'un réseau hydrographique navigable, les échanges reposaient essentiellement sur les transports terrestres. Les routes empruntaient des vallées encaissées et devaient franchir des cols. Elles nécessitaient un entretien constant et coûteux. De plus, l'enneigement entravait la circulation durant l'hiver.

Les luttes constantes entre villes voisines mais aussi avec les seigneurs des environs, tel que le marquis de Santillana, le marquis d'Aguilar et le comte de Salinas, pour l'exercice de la juridiction terrestre et maritime constituèrent un autre frein important au rayonnement des Quatre Villes. Laredo et Castro Urdiales se disputèrent le contrôle de l'embouchure de l'Agüera, secteur stratégique par lequel le fer de l'arrière-pays destiné à l'exportation gagnait la mer ${ }^{43}$. Bien que Laredo ait été la plus importante des quatre villes portuaires cantabres, elle ne parvint pas à s'imposer aux trois autres. Chacune contrôlait une zone d'influence propre qui ne s'étendait guère au-delà de sa juridiction terrestre (término municipal) et des eaux maritimes situées en avant de la côte ${ }^{44}$. De fait elles subissaient l'influence de Bilbao, centre économique d'une tout autre importance, qui se faisait sentir jusqu'en mer. En 1544, la municipalité de Castro Urdiales se plaignit des pilotes de Bilbao et de Portugalete qui utilisaient les facilités offertes par le " port et conche " de Castro Urdiales pour se porter ensuite à la rencontre des navires et les détourner, avec leurs marchandises, vers leur port d'origine $^{45}$.

Une partie du fer produit par les ferrons cantabres était captée par des marchands biscayens qui venaient l'acheter sur place dans les villes de la côte. Le cas du marchand de Bilbao Martín de Arana est significatif. Dans la première décennie du $\mathrm{XVI}^{\mathrm{e}}$ siècle il conduit vers Bilbao de grandes quantités de fer Cantabre. En 1506, il fait charger 150 quintaux sur une pinasse stationnant sur l'Agüera. Les opérations de ce type se succèdent au cours des années suivantes ${ }^{46}$. En 1509, il envoie trois embarcations prendre

43. Archivo de la Real Chancillería de Valladolid, Pleitos Civiles, Zarandona y Wals, subserie olvidados, sig. c $86-2 \mathrm{f}^{\circ} 1 \mathrm{v}^{\circ}-2 \mathrm{r}^{\circ}, 1381$, procès lors duquel les habitants de Laredo accusent leurs voisins de Castro Urdiales d'entraver la circulation des personnes et des biens sur le fleuve Agüera depuis 1376. Le juge trancha en faveur de Castro Urdiales parce que le notaire de Laredo avait falsifié des témoignages.

44. ARízAga Bolumburu, Beatriz, "La villa de Laredo y sus términos jurisdiccionales ", Historica et Philologica, Santander, 2002, p. 183-195; FERnÁndEz GonZÁlez, Lorena, Santander..., op. cit., p. 153-168.

45. Baró Pazos, Juan, Galván Rivero, Carmen, Libro de Ordenanzas. op. cit., p. 174-175, " ocurren, en que los lemanes de la villa de Portogalete, e canal de la villa de Bilvao, e de otras partes bengan a esta villa de Castro, e lleben las naos e navíos que a ella bienen [...] antes que surgan en el puerto e cocha desta dicha villa [...] E aunque las tales naos e navíos quieren descargar e bender las dichas mercaderías, los dichos lemanes no los dexan, e los lleban a la canal de Bilvao ".

46. Blanco Campos, Emma, Álvarez Llopis, Elisa, García de Cortázar, José Ángel, Libro del Concejo..., op. cit., doc. 15. En 1500 Martín de Arana transporte 150 quintaux (p. 335, 
livraison de 500 quintaux de fer. Pour remonter les fleuves côtiers il utilisait en général des chaloupes de faible tirant d'eau. Chacune pouvait charger en moyenne 150 quintaux de fer, ce qui représentait sur la base de $46 \mathrm{~kg}$ par quintal une charge utile de 7500 kg environ. Pour des raisons de sécurité au regard des autres navires fréquentant le port, elles devaient charger et décharger à part, en dehors des quais ${ }^{47}$. Les marchands de Bilbao avaient ainsi mis la main sur le transport du minerai de fer comme sur la vente du produit semi-fini et contrôlaient ainsi le commerce dont le gros des flux passait par Bilbao. Le seul marchand anglais qu'on aperçoit dans les sources écrites figure dans les livres de comptes de la municipalité de Castro Urdiales en 1507, année durant laquelle Bilbao fut désolé par une épidémie de peste ${ }^{48}$.

Les céréales représentaient un poste important des importations. Elles provenaient en premier lieu de la Tierra de Campos, au sud de la cordillère cantabrique, mais en période de pénurie il fallait en importer de France, d'Angleterre et de Flandre. À partir du XvI ${ }^{\mathrm{e}}$ siècle les importations en provenance d'Andalousie se développèrent pour compenser la rupture d'approvisionnement liée à la guerre avec la France ${ }^{49}$.

À partir du Xv $\mathrm{V}^{\mathrm{e}}$ siècle, les conseils municipaux cherchèrent à améliorer les accès terrestres et maritimes de leur ville en construisant et en faisant réparer les chemins, les ponts et les quais ${ }^{50}$. En 1428 Santander disposait d'un quai des tonneliers (cay de los toneleros) ${ }^{51}$. En 1489, la municipalité de Laredo constatant que le quai existant était trop petit et que les marchands se détournaient vers d'autres ports fit entreprendre des travaux d'agrandissement. La ville consentit à faire de lourdes dépenses, estimées à 6000 doublons et qui furent en grande partie financées par des impôts (sisas) ${ }^{52}$. San

$\mathrm{f}^{\circ} 133$ ); en 1508 ce sont 130 quintaux en mars et 260 en mai (p. $366, \mathrm{f}^{\circ} 151$ et p. $388, \mathrm{f}^{\circ} 166$ ); en 1509492 quintaux (p. 395, f ${ }^{\circ} 170$ ); en 1510 il transporte 95 quintaux à Bilbao et 100 à Laredo (p. 410, f 181 et p. 411, $\mathrm{f}^{\circ} 181$ ).

47. Baró Pazos, Juan, Galván Rivero, Carmen, Libro de Ordenanzas..., op. cit., p. 178, $\mathrm{f}^{\circ} 63$ $\mathrm{v}^{\circ}-64$, dispositions concernant les châtaigniers.

48. Blanco Campos, Emma, Álvarez Llopis, Elisa, García de CortÁzar, José Ángel, Libro del concejo..., op. cit., doc. 15, p. 360. Un dénommé Huarta, marchand anglais, demande une licence pour exporter 100 quintaux de fer.

49. Le blé parvenait dans les Quatre Villes grâce aux contacts commerciaux de villes plus actives comme Bilbao et Saint-Sébastien. AGS, Cámara de Castilla. Pueblos. Leg. 17, Mayo 16 1504. SuÁREZ FERnÁndez, Luis, Política internacional de Isabel la Católica, t. II, 1482-1488, doc. 97, p. 400. SolóRZANo TelecheA, Jesús Ángel, Colección diplomática..., op. cit., doc. 133, p. 179. Отте, Enrique, Sevilla y sus mercaderes a fines de la Edad Media, Sevilla, 1996, p. 257.

50. SOLÓRZANO TELECHEA, Jesús Ángel, AÑíBARRo RoDRíGuEZ, Javier, « Infraestructuras e instalaciones portuarias, fluviales e hídricas en las villas del norte peninsular a finales de la Edad Media : las obras públicas como instrumentos del poder ", Musulmanes y Cristianos frente al agua en las ciudades medievales, Santander, 2008, p. 273-305.

51. FERnÁNDEZ gONZÁLEZ, Lorena, Archivo de la Catedral de Santander, Santander, 1994, doc. 212 .

52. Blanco Campos, Emma, Álvarez llopis, Elisa, García de Cortázar, José Ángel, Documentación referente a Cantabria..., op. cit., doc. 893, p. 537, " las cargas y descargas 
Vicente de la Barquera disposait d'un quai de pierre de 100 brasses de long, qui nécessita des réparations en $1506^{53}$. La ville devait en outre réparer les deux ponts, l'un de 200 brasses, l'autre de 50 brasses, qui, enjambant les deux rias qui l'encadraient, lui permettaient de communiquer avec le reste de la province ${ }^{54}$. Ces efforts ne suffirent pas à rendre ces divers ports suffisamment attractifs par rapport à Bilbao.

Tout en contribuant à animer l'économie régionale de la Cantabrie, les Quatre Villes de la Côte de la Mer, dont les activités étaient largement tournées vers la mer, jouaient cependant un rôle secondaire à l'échelle de la façade maritime du nord de la Castille aux XIV et $\mathrm{Xv}^{\mathrm{e}}$ siècles. Ce relatif effacement, qui caractérise aussi les Asturies et qui contraste avec l'insertion beaucoup plus grande dans les échanges maritimes internationaux des ports galiciens à l'ouest, biscayens et guipúzcoans à l'est, a déjà été souligné par Elisa Ferreira Priegue ${ }^{55}$. Une analyse plus détaillée des sources disponibles pour la Cantabrie, tout en confirmant cette appréciation générale, montre en outre une relative dépendance économique par rapport à Bilbao. À partir de sa position au fond de la ria du Nervión, Bilbao avait étendu son influence aux ports immédiatement voisins : Portugalete, Plencia, Castro Urdiales. Son rôle dans la structuration de l'espace était assez fort pour lui permettre de capter l'essentiel des trafics de marchandises aux dépens de villes portuaires pourtant situées à une certaine distance de lui, comme Laredo et Santander en Cantabrie ou Bermeo en Biscaye, ainsi que le montrent les circuits d'exportation de la laine castillane. Tout en s'affirmant comme un élément clé dans la hiérarchie portuaire de la côte nord-péninsulaire, le rôle central de Bilbao ne peut se comprendre sans la présence de ports secondaires qui concourraient à sa prospérité ${ }^{56}$. Cela conduit donc à relativiser des approches entièrement centrées sur un port ou sur une province historique et à privilégier dans la mesure du possible les ensembles économiques tels qu'ils fonctionnaient en jouant sur les échelles spatiales et en ne se laissant pas enfermer dans des cadres politiques ou administratifs trop stricts.

de las dichas flotas, por no ser seguras, las dichas naos en los tiempos de fortunas y por se a ver algunas de ellas perdido con sus mercaderias con algunas partes de las gentes que en ellas estavan, han çesado y dejado la dicha contratación y se fueron a otras pares donde el contrato de la villa es pedido "; AGS, Cámara de Castilla, Memoriales, leg. 148, doc. 9.

53. AGS, Cámara de Castilla, Pueblos, leg. 17, doc. 348, 12 Junio $1506, \mathrm{f}^{\circ} 1 \mathrm{v}^{\circ}$ : « tiene neçesidad de remediar un muelle de piedra que está todo caydo en la rybera de la mar de más de çien braças".

54. Blanco Campos, Emma, Álvarez llopis, Elisa, García de Cortázar, José Ángel, Documentación referente a Cantabria..., op. cit., doc. 589, p. 370; AGS, Cámara de Castilla, Memoriales, leg. 120, doc. 80. Voir aussi AGS, Registro General del Sello, Abril 1500, $\mathrm{f}^{\circ} 211$.

55. FERREIRA PRIEGUE, Elisa, "Las rutas marítimas y comerciales del flanco ibérico desde Galicia hasta Flandes ", El fuero de San Sebastián y su época, San Sebastián, 1981, p. 217-234.

56. BоснасA, Michel, "Typologie et fonctions des ports du Bordelais (fin Xve-début xvI ${ }^{\mathrm{e}}$ siècle), Historia, Instituciones, Documentos, t. 35, 2008, p. 63-83. 